\title{
Guest editorial-special issue on service modeling and design for the future Internet
}

\author{
Fabrizio Granelli • Michael Devetsikiotis
}

Published online: 12 August 2011

(C) The Brazilian Computer Society 2011

Today, the Internet represents the major communication and collaboration infrastructure worldwide. However, its value is defined by its services.

While enthusiasm of developers in proposing novel services is reshaping the current Internet, there is a body of work that is examining issues related to network-level as well as application and service-layer topics of analysis, design, monitoring, and experimentation. Indeed, top-down interplay between services and networking creates unique modeling, design, and implementation challenges. The coupling of the above two aspects is required to enable the evolution of the Internet into the Internet of the Future, a pervasive cyberspace providing humanity with an unprecedented capability to communicate, share knowledge, and evolve.

In this context, the purpose of this special issue is to focus the community's efforts in building up this important area, by discussing associated issues and required breakthroughs in research and development.

The selected papers provide the readers with a snapshot of the recent and most interesting developments in modeling and design methodologies for the services of the Future Internet. The papers were selected from preliminary works presented at the IEEE Workshop on Computer-Aided Modeling, Analysis and Design of Communication Links and Networks (CAMAD 2010), and the IEEE Workshop on En-

F. Granelli (凶)

Department of Information Engineering and Computer Science, University of Trento, via Sommarive 14, 38123 Trento Italy e-mail: granelli@disi.unitn.it

\section{Devetsikiotis}

Department of Electrical and Computer Engineering, North Carolina State University, Raleigh, NC, USA

e-mail: mdevets@ncsu.edu abling the Future Service Oriented Internet (EFSOI 2010), both held at the end of 2010 .

The following paragraphs provide a brief description of the papers and their contributions.

In "Automated Transport Service Management in the Future Internet: Concepts and Operations," Callegati, Campi, and Cerroni describe an approach to providing advanced communication services for the Future Internet. The proposed solution is based on the usage of a Cognitive Transport Service layer on top of the traditional transport layer, used by the network to automatically negotiate the required transport service with the applications.

Cloud computing represents an emerging computing paradigm and this is the focus of the following paper, "Cloud Computing Services: Taxonomy and Comparison," by Höfer and Karagiannis. The paper examines the available cloud computing services and proposes a tree-structured taxonomy to enable classification and comparison of the different cloud computing services. The taxonomy is explained and verified using existing cloud services as examples.

The novel concept of an Application Delivery Platform (ADP) is presented by Menkens and Wuertinger in the paper "From Service Delivery to integrated SOA based Application Delivery in the Telecommunication Industry." An Application Delivery Platform extends the concept of an SDP by broadening the scope from reusable SOA services over an application architecture to end user applications on client platforms and devices. The paper focuses on evaluating the requirements of different stakeholders and fulfilling them through combining concepts of Service Oriented Architecture (SOA) hosting and deployment platforms with open telecommunication SDP architecture concepts.

A different perspective is presented by Stai, Karyotis, and Papavassiliou in "A Socially-driven Topology Improvement Framework with Applications in Content Distribution 
and Trust Management," where a distributed and dynamic physical topology modification framework is proposed by exploiting the power varying capabilities of multihop wireless networks and inspired by social structures of the higher protocol layers. The paper provides a demonstration of the application of social features to network and service design.

Finally, video transmission services are analyzed in "Packet Level Video Quality Evaluation of Extensive H.264/ AVC and SVC Transmission Simulation" by Skupin, Hellge, Schierl, and Wiegand. The paper tackles the problem of evaluating the performance of video delivery of error prone channels by means of simulation, where fast simulation is required to enable effective estimation of the impact of de- sign parameters on the users' perceived quality. The proposed approach significantly reduces the overall simulation time by eliminating redundancy in the evaluation phase and utilizing the prediction structure of the video codec.

We hope the issue will provide a useful reference to the current state of the research in modeling and design of services for the Future Internet, and we would like to thank the authors for their contributions, the anonymous reviewers for their hard work to support them with constructive comments to improve the papers, as well as the Editors-in-Chief of the Journal and their associated editorial team for their support.

Guest Editors 